\title{
A REMARK ON ZETA FUNCTIONS ${ }^{1}$
}

BY

\author{
JUN-ICHI IGUSA
}

Dedicated to Professor Kenkichi Iwasawa on his 60th birthday

\begin{abstract}
In the adelic definition of the zeta function by Tate and Iwasawa, especially in the form given by Weil, one uses all Schwartz-Bruhat functions as "test functions"; we have found that such an adelic zeta function relative to $Q$ contains the Dedekind zeta function of any finite normal extension of $Q$ and that the normality assumption can be removed if Artin's conjecture is true.
\end{abstract}

Introduction. We shall first review the definition of the zeta distribution associated with a number field $K$ : let $\mathbf{A}_{K}$ resp. $\mathbf{A}_{K}^{\times}$denote the adele resp. idele groups of $K, d^{\times} x$ a Haar measure on $\mathbf{A}_{K}^{\times}$, and $\delta\left(\mathbf{A}_{K}\right)$ the Schwartz-Bruhat space of the locally compact additive group $\mathbf{A}_{K}$; the topological dual $\delta\left(\mathbf{A}_{K}\right)^{\prime}$ of $\delta\left(\mathbf{A}_{K}\right)$ is then the space of tempered distributions on $\mathbf{A}_{K}$. Let $|x|$ denote the modulus of an idele $x$; then

$$
\Delta_{K}(s)[\Phi]=\int_{\mathbf{A}_{K}^{\times}} \Phi(x)|x|^{s} d^{\times} x
$$

defines the zeta distribution on $\mathbf{A}_{K}$, which is an $\delta\left(\mathbf{A}_{K}\right)^{\prime}$-valued holomorphic function in $\operatorname{Re}(s)>1$ and it has a meromorphic continuation to the whole complex $s$-plane with simple poles at $s=0,1$ and satisfies the well-known functional equation. Furthermore if we take the "standard function" $\Phi_{0}$ on $A_{K}$ as $\Phi$, then we get

$$
\Delta_{K}(s)\left[\Phi_{0}\right]=\operatorname{const}\left(2^{-r_{2}} \pi^{-n / 2}\right)^{s} \Gamma\left(\frac{1}{2} s\right)^{r_{1}} \Gamma(s)^{r_{2}} \zeta_{K}(s),
$$

in which $r_{1}$, resp. $r_{2}$, are the numbers of real, resp. complex, conjugates of $K$, $n=r_{1}+2 r_{2}=[K: \mathbf{Q}]$, and $\zeta_{K}(s)$ is the Dedekind zeta function of $K$. We refer to [12] for the details; we recall that in op. cit. Weil gave a "reinterpretation" of the work of Tate [9], independently of Iwasawa, on Hecke's zeta functions. We further recall that we have only one choice for $A>0$ so that

$$
\Lambda_{K}(s)=A^{s} \Gamma\left(\frac{1}{2} s\right)^{r_{1}} \Gamma(s)^{r_{2}} \zeta_{K}(s)
$$

becomes invariant under $s \rightarrow 1-s$; and the unique $A$ is given by

Received by the editors November 1, 1977.

AMS (MOS) subject classifications (1970). Primary $12 \mathrm{A70}$.

${ }^{1}$ This work was partially supported by the National Science Foundation. 


$$
A=2^{-r_{2}} \pi^{-n / 2}|d|^{1 / 2},
$$

in which $d$ is the discriminant of $K$; cf. [5].

We shall explain the content of this paper: we take $Q$ as $K$ and $\Phi_{f} \otimes \Phi_{\infty}$ as $\Phi$, in which $\Phi_{f}$ is the finite part of the standard function on $A_{Q}$, i.e., the characteristic function of the product of all $\mathbf{Z}_{p}$, and $\Phi_{\infty}$ is, for a moment, an arbitrary Schwartz function on $\mathbf{Q}_{\infty}=\mathbf{R}$; we normalize the Haar measure $d^{\times} x$ on $\mathbf{A}_{\mathbf{Q}}^{\times}$in the usual manner so that we get

$$
\Delta_{\mathbf{Q}}(s)\left[\Phi_{f} \otimes \exp \left(-\pi x_{\infty}^{2}\right)\right]=\Lambda_{\mathbf{Q}}(s) .
$$

Then every number field $K$ determines an even Schwartz function $F_{K}$ on $\mathbf{R}$ satisfying

$$
\Delta_{\mathbf{Q}}(s)\left[\Phi_{f} \otimes F_{K}\right]=\Lambda_{K}(s)
$$

provided that $\zeta_{K}(s) / \zeta_{\mathbf{Q}}(s)$ is an entire function; we recall that this condition is satisfied if $K$ is normal over $\mathbf{Q}$ and that it is always satisfied if Artin's conjecture is true; cf. [1], [2], [3]. We can therefore say, more suggestively, that the zeta function (multiplied by the usual gamma factors) of an arbitrary finite (normal) extension of $\mathbf{Q}$ can be obtained as a "special value" of the zeta distribution $\Delta_{\mathbf{Q}}(s)$ on $\mathbf{A}_{\mathbf{Q}}$. We might also say that $F_{\mathbf{Q}}\left(x_{\infty}\right)=\exp \left(-\pi x_{\infty}^{2}\right)$ is the first member of the infinite sequence $\left\{F_{K}\right\}_{K}$ of functions $F_{K}$ on $\mathbf{R}$ indexed by the set of all finite (normal) extensions $K$ of $\mathbf{Q}$. We have examined some properties of $F_{K}$ in the general case and given an explicit expression for $F_{K}\left(x_{\infty}\right)$ in the case where $K$ is a quadratic field.

1. Some lemmas. We shall recall some lemmas on the classical Mellin transformation; for the sake of simplicity we shall denote a real variable (which will later become a complex variable) by $x$ instead of $x_{\infty}$ as in the Introduction. Let $F$ denote a continuous function on $\mathbf{R}_{+}^{\times}$, the multiplicative group of positive real numbers, such that $F(x) x^{s}$, where $s$ is restricted as $\operatorname{Re}(s)>\sigma_{0}$ for some $\sigma_{0}$, is absolutely integrable with respect to $d \log x$; then

$$
Z(s)=\int_{0}^{\infty} F(x) x^{s} d \log x
$$

defines a holomorphic function $Z$ in $\operatorname{Re}(s)>\sigma_{0}$. We call $Z$ the Mellin transform of $F$ and the correspondence $F \rightarrow Z$ the Mellin transformation. For instance $\Gamma(s)$ is the Mellin transform of $\exp (-x)$; and as in that case $Z(s)$ often has a meromorphic continuation to the whole $s$-plane. We observe that if $Z$ is the Mellin transform of $F$, then $a^{-s} Z(s)$, resp. $a^{-1} Z\left(a^{-1} s\right)$, for any $a>0$ are the Mellin transforms of $F(a x)$, resp. $F\left(x^{a}\right)$. In the following we put $\sigma=\operatorname{Re}(s), t=\operatorname{Im}(s)$ so that we can write $s=\sigma+t i$; the following lemma plays a crucial role in this paper:

LEMMA 1. Let $(F)$ denote the space of even Schwartz functions on $\mathbf{R}$ and $(Z)$ 
the space of meromorphic functions $Z(s)$ on the s-plane with simple poles at most at $s=0,-2,-4, \ldots$ and such that $P(s) Z(s)$ for any polynomial $P(s)$ tends uniformly to 0 as $|t| \rightarrow \infty$ in any strip $\sigma_{1} \leqslant \sigma \leqslant \sigma_{2}$ of finite width: then the Mellin transformation gives a C-linear bijection of $(F)$ to $(Z)$. Furthermore if

$$
F(x) \approx \sum_{k=0}^{\infty} c_{k} x^{2 k}
$$

is the Maclaurin expansion of $F(x)$ in $(F)$ and $Z(s)$ is the corresponding function in $(Z)$, then

$$
c_{k}=\operatorname{Res}_{s=-2 k} Z(s)=\lim _{s \rightarrow-2 k}(s+2 k) Z(s)
$$

for $k=0,1,2, \ldots$

This follows, e.g., from Proposition 3 in $[6$, p. 117] in view of the fact that (F) can be identified with the space of functions of type $\left(F_{2}\right)$ with $\{0,2,4, \ldots\}$ as the sequence $\left\{\lambda_{k}\right\}_{k}$ (in that paper) and with $m_{k}=1$ for $k=1,2,3, \ldots$

We shall use various properties of $\Gamma(s)$; of these we shall mention just one as a lemma:

LEMMA 2. We have

$$
|\Gamma(s)|=(2 \pi)^{1 / 2}|t|^{\sigma-1 / 2} \exp \left(-\frac{1}{2} \pi|t|\right)(1+o(1)),
$$

in which $o(1)$ denotes a quantity which tends uniformly to 0 as $|t| \rightarrow \infty$ in any strip $\sigma_{1}<\sigma \leqslant \sigma_{2}$ of finite width.

We refer to [7, §2, pp. 307-309] for the proof. In the following lemma $x$ is a complex variable:

LEMMA 3. For any given $\theta_{0}>0$ let $\mathcal{F}$ denote the space of holomorphic functions $F(x)$ in the angular region $|\arg x|<\theta_{0}$ (of the universal covering space of $\left.\mathbf{C}^{\times}\right)$such that $|x|^{a} F(x)$ is bounded if $a$, $x$ are restricted as $\sigma_{1}<a<\sigma_{2}$, $|\arg x|<\theta_{0}-\varepsilon$ for every $\varepsilon>0$ and $1<\sigma_{1}<\sigma_{2}<\infty$; let $\mathcal{Z}$ denote the space of holomorphic functions $Z(s)$ in $\operatorname{Re}(s)>1$ such that $\exp (\theta t) Z(s)$ is bounded if $\theta$, $s$ are restricted as $|\theta| \leqslant \theta_{0}-\varepsilon, \sigma_{1} \leqslant \sigma \leqslant \sigma_{2}$ for the same $\varepsilon, \sigma_{1}, \sigma_{2}$ as above. Then the Mellin transformation gives a $\mathbf{C}$-linear bijection of $\mathscr{F}$ to $\mathcal{Z}$.

This is a special case of the "reciprocity" in Mellin (op. cit., §8, pp. 318-324).

We shall change the subject: let $f(s)$ denote a meromorphic function on the $s$-plane (different from the constant 0 ); then it has an order, ord $(f)$, satisfying $0<\operatorname{ord}(f) \leqslant \infty, \operatorname{ord}(1 / f)=\operatorname{ord}(f)$. If $f_{1}(s), f_{2}(s)$ are two meromorphic functions, then 


$$
\operatorname{ord}\left(f_{1} f_{2}\right) \leqslant \max \left\{\operatorname{ord}\left(f_{1}\right), \operatorname{ord}\left(f_{2}\right)\right\} ;
$$

in view of $\operatorname{ord}\left(1 / f_{2}\right)=\operatorname{ord}\left(f_{2}\right)$ the left-hand side can be replaced by $\operatorname{ord}\left(f_{1} / f_{2}\right)$. Furthermore if $f(s)$ is an entire function of finite order, then $\lambda=\operatorname{ord}(f)$ is the smallest nonnegative real number such that

$$
\log |f(s)| \leqslant M_{\varepsilon} \cdot|s|^{\lambda+\varepsilon}
$$

for $|s| \geqslant 1$, in which $\varepsilon>0$ is arbitrary and $M_{\varepsilon}$ is a constant depending on $\varepsilon$. For instance $1 / \Gamma(s)$ is an entire function of order 1 . We refer to [8, Chapter VIII] for a general theory.

We recall that $s(1-s) \Lambda_{K}(s)$ for any number field $K$ is an entire function of order 1 and is invariant under $s \rightarrow 1-s ;$ cf. [5], [11]. Therefore if we put

$$
E_{K}(s)=\Lambda_{K}(s) / \Lambda_{\mathbf{Q}}(s),
$$

then $E_{K}(s)$ is a meromorphic function of order at most 1 , and it is invariant under $s \rightarrow 1-s$.

2. The function $F_{K}$. We shall use the same notation as before: the problem is to examine the solvability of the equation $\Delta_{\mathbf{Q}}(s)\left[\Phi_{f} \otimes \Phi_{\infty}\right]=\Lambda_{K}(s)$ in $\Phi_{\infty}$, which is an unknown even Schwartz function on $\mathbf{R}$. If we put

$$
Z_{K}(s)=\Lambda_{K}(s) / 2 \zeta_{\mathbf{Q}}(s)=\frac{1}{2} \pi^{-s / 2} \Gamma\left(\frac{1}{2} s\right) E_{K}(s),
$$

then the above equation can be rewritten as

$$
\int_{0}^{\infty} \Phi_{\infty}(x) x^{s} d \log x=Z_{K}(s)
$$

we shall examine this equation by using Lemma 1 :

Suppose that the equation is solvable; then $Z_{K}$ belongs to the space $(Z)$ in Lemma 1. The relation between $Z_{K}(s)$ and $E_{K}(s)$ then shows that $E_{K}(s)$ is an entire function. Since $1 / \Gamma(s)$ is an entire function, by using the duplication formula

$$
\Gamma(s)=2^{s-1} \pi^{-1 / 2} \Gamma\left(\frac{1}{2} s\right) \Gamma\left(\frac{1}{2}(s+1)\right)
$$

if $r_{1}=0$, we see that $\zeta_{K}(s) / \zeta_{Q}(s)$ is an entire function.

Conversely suppose that $\zeta_{K}(s) / \zeta_{\mathbf{Q}}(s)$ is an entire function; then $E_{K}(s)$ is holomorphic in $\sigma>0$. Since $E_{K}(s)$ is invariant under $s \rightarrow 1-\mathrm{s}$, it is also holomorphic in $\sigma<1$; hence $E_{K}(s)$ is an entire function. This implies that $Z_{K}(s)$ is a meromorphic function with simple poles at most at $s=0,-2$, $-4, \ldots$ On the other hand, by using the duplication formula again if $r_{1}=0$, we see that $E_{K}(s)$ is bounded on $\operatorname{Re}(s)=\sigma$ for any $\sigma>1$; by the invariance of $E_{K}(s)$ under $s \rightarrow 1-s$ it is also bounded on $\operatorname{Re}(s)=\sigma$ for any $\sigma<0$. Furthermore we have

$$
\log \left|E_{K}(s)\right| \leqslant M_{\varepsilon} \cdot|s|^{1+\varepsilon}
$$

for $|s| \geqslant 1$, in which $M_{\varepsilon}$ is a constant depending on $\varepsilon>0$. Therefore the 
standard application of Phragmén-Lindelöf's theorem to $E_{K}(s)$ shows that it is bounded in any strip $\sigma_{1} \leqslant \sigma \leqslant \sigma_{2}$ of finite width. Consequently if $P(s)$ is a polynomial in $s$, then by Lemma 2 we will have

$$
\left|P(s) Z_{K}(s)\right| \leqslant \text { const }\left|P(s) \Gamma\left(\frac{1}{2} s\right)\right|=o(1)
$$

as $|t| \rightarrow \infty$ in any such strip. Therefore $Z_{K}$ is in $(Z)$; hence by Lemma 1 the equation is solvable. We have thus completed the proof of the following theorem:

THEOREM 1. Let $K$ denote an arbitrary number field and $\Phi_{f}$ the characteristic function of the product of all $\mathbf{Z}_{p}$; then the equation $\Delta_{\mathbf{Q}}(s)\left[\Phi_{f} \otimes \Phi_{\infty}\right]=\Lambda_{K}(s)$ is uniquely solvable by an even Schwartz function $\Phi_{\infty}$ on $\mathbf{R}$ if and only if $\zeta_{K}(s) / \zeta_{\mathbf{Q}}(s)$ or, equivalently, $E_{K}(s)$ is an entire function.

We have already mentioned in the Introduction that $\zeta_{K}(s) / \zeta_{Q}(s)$ is an entire function if $K$ is normal over $\mathbf{Q}$ and that it is always an entire function if Artin's conjecture is true. In the following we shall proceed under the assumption that $\zeta_{K}(s) / \zeta_{Q}(s)$ is an entire function: if we denote the unique $\Phi_{\infty}$ in Theorem 1 by $F_{K}$, then we have $F_{\mathrm{Q}}(x)=\exp \left(-\pi x^{2}\right)$; we shall summarize some properties of $F_{K}$ in the following theorem:

THEOREM 2. The function $F_{K}$ is real-valued, is equal to its Fourier transform:

$$
\int_{-\infty}^{\infty} F_{K}(y) \mathbf{e}(x y) d y=F_{K}(x)
$$

where $\mathrm{e}(x)=\exp (2 \pi i x)$, and has the following Maclaurin expansion:

$$
F_{K}(x) \approx \sum_{k=0}^{\infty} E_{K}(2 k+1) \cdot\left(-\pi x^{2}\right)^{k} / k !
$$

in which

$$
F_{K}(0)=E_{K}(1)=(2 \pi)^{-r_{2}}|d|^{1 / 2} \cdot \operatorname{Res}_{s=1} \zeta_{K}(s) .
$$

The radius of convergence of this power series depends on $n=[K: Q]$; it is $\infty$, $|d|^{-1 / 2}$, or 0 according as $n=1, n=2$, or $n \geqslant 3$. Furthermore $F_{K}(x)$ has $a$ holomorphic continuation to $\operatorname{Re}(x) \neq 0$; it even has a holomorphic continuation beyond $\operatorname{Re}(x)=0, x \neq 0$ if $n \geqslant 3$.

Proof. We observe that $x^{s}$ and $Z_{K}(s)$ take complex-conjugate values at complex-conjugate variables; hence $F_{K}(s)$ is real-valued. If $\psi$ is a nontrivial character of $\mathbf{A}_{\mathbf{Q}} / \mathbf{Q}$ and if we define the Fourier transformation $\Phi \rightarrow \Phi^{*}$ in $\delta\left(\mathbf{A}_{\mathbf{Q}}\right)$ with respect to the bicharacter $\psi(x y)$ and the corresponding autodual measure, then we will have $\Delta_{Q}(s)\left[\Phi^{*}\right]=\Delta_{Q}(1-s)[\Phi]$ for every $\Phi$. We take $\Phi_{f}$ $\otimes F_{K}$ as $\Phi$ and, at the same time, normalize $\psi$ or rather its local components as $\psi_{p}\left(x_{p}\right)=\mathrm{e}\left(-\right.$ fractional part of $\left.x_{p}\right)$ for every $p, \psi_{\infty}\left(x_{\infty}\right)=\mathrm{e}\left(x_{\infty}\right)$; then, by using $\Lambda_{K}(s)=\Lambda_{K}(1-s)$, we see that $F_{K}$ and its Fourier transform have the 
same Mellin transform. Therefore $F_{K}$ is equal to its Fourier transform.

According to Lemma 1 we have

$$
F_{K}(x) \approx \sum_{k=0}^{\infty} \operatorname{Res}_{s=-2 k} Z_{K}(s) \cdot x^{2 k}
$$

in which

$$
\begin{aligned}
\operatorname{Res}_{s=-2 k} Z_{K}(s) & =\frac{1}{2} \pi^{k} E_{K}(-2 k) \cdot \operatorname{Res}_{s=-2 k} \Gamma\left(\frac{1}{2} s\right) \\
& =\pi^{k} E_{K}(2 k+1) \cdot(-1)^{k} / k !
\end{aligned}
$$

for $k=0,1,2, \ldots$; the expression for $E_{K}(1)$ follows from the definition of $E_{K}(s)$.

We shall determine the radius of convergence of the Maclaurin series: if $s \rightarrow \infty$ along the real axis, then by Stirling's formula we have

$$
\log \Gamma(s)=s \log s-s-\frac{1}{2} \log s+O(1)
$$

we also have $\zeta_{K}(s) / \zeta_{\mathbf{Q}}(s) \rightarrow 1$. Therefore we get

$$
\begin{aligned}
& (1 / 2 k) \log \left|E_{K}(2 k+1) \pi^{k} / k !\right| \\
& \quad=\frac{1}{2}(n-2)(\log (k / \pi)-1)+\log |d|^{1 / 2}+o(1)
\end{aligned}
$$

as $k \rightarrow \infty$; hence by Cauchy's formula the radius of convergence is $\infty$, $|d|^{-1 / 2}$, or 0 according as $n=1, n=2$, or $n \geqslant 3$.

Finally we put $\theta_{0}=\frac{1}{4} \pi n$; we observe that $A^{s} \zeta_{K}(s) / \zeta_{Q}(s)$ is bounded in any strip $\sigma_{1}<\sigma \leqslant \sigma_{2}$ if $1<\sigma_{1} \leqslant \sigma_{2}<\infty$. Therefore for any small $\varepsilon>0$ we have

$$
\left|\exp (\theta t) Z_{K}(s)\right| \leqslant \text { const } \exp \left(\left(\theta_{0}-\varepsilon\right)|t|\right)\left|\Gamma\left(\frac{1}{2} s\right)^{r_{1}} \Gamma(s)^{r_{2}}\right|
$$

if $\theta, s$ are restricted as $|\theta| \leqslant \theta_{0}-\varepsilon, \sigma_{1} \leqslant \sigma \leqslant \sigma_{2}$; and by Lemma 2 the right-hand side is bounded. Since $Z_{K}$ is the Mellin transform of $F_{K}$, we see by Lemma 3 that $F_{K}(x)$ is holomorphic in the angular region $|\arg x|<\theta_{0}$. If we exclude the known case where $n=1$, we will have $\theta_{0}>\frac{1}{2} \pi$; we even have $\theta_{0}>\frac{1}{2} \pi$ if $n \geqslant 3$. This completes the proof.

We might mention that in [4] Hardy and Titchmarsh characterized the subspace of $L^{2}\left(\mathbf{R}_{+}^{\times}, d x\right)$ consisting of all $f(x)$ satisfying

$$
f(x)=\left(2 \pi^{-1}\right)^{1 / 2} \cdot \int_{0}^{\infty} \cos (x y) f(y) d y
$$

the condition they found is that $f(x)$ be of the form

$$
f(x)=(1 / 2 \pi i) \int_{1 / 2-\infty i}^{1 / 2+\infty i} \phi(s) x^{-s} d s
$$

in which $\phi\left(\frac{1}{2}+t i\right)$ is in $L^{2}(\mathbf{R}, d t)$ and satisfies the following functional equation: 


$$
\phi(s)=\left(2 \pi^{-1}\right)^{1 / 2} \Gamma(s) \cos \left(\frac{1}{2} \pi s\right) \phi(1-s) .
$$

Among their examples of $\operatorname{such} f(x)$ we find $\exp \left(-\frac{1}{2} x^{2}\right)$ and $\operatorname{sech}\left(\frac{1}{2} \pi\right)^{1 / 2} x$; in our notation they are, respectively, $F_{Q}\left((2 \pi)^{-1 / 2} x\right)$ and $4 \cdot F_{K}\left((2 \pi)^{-1 / 2} x\right)$ for $K=\mathbf{Q}(i)$.

3. $F_{K}$ for $[K: Q]=2$. We shall determine the function $F_{K}$ in the special case where $[K: \mathbf{Q}]=2$. We have $\zeta_{K}(s)=\zeta_{\mathbf{Q}}(s) L(s)$, in which

$$
L(s)=\sum_{n=1}^{\infty}(d / n) n^{-s}
$$

is the $L$-series associated with the special Dirichlet character $(d / n)$ with conductor $|d|$ called Kronecker's symbol; we shall use the following classical lemma:

LEMMA 4. For any integer $m$ we have

$$
\sum_{n \bmod d}(d / n) \mathbf{e}(m n /|d|)=(d / m) d^{1 / 2},
$$

in which $d^{1 / 2}=|d|^{1 / 2}$ if $d<0$.

We refer to [10, pp. 328-329] for the proof. We shall also use Abel's inequality which can be stated as follows: let

$$
a_{0}, a_{1}, \ldots, a_{n}, b_{0}, b_{1}, \ldots, b_{n}, c_{0}, c_{1}, \ldots, c_{n}
$$

denote not necessarily real numbers satisfying the following conditions:

$$
\begin{aligned}
& \left|a_{0}\right|,\left|a_{0}+a_{1}\right|, \ldots,\left|a_{0}+a_{1}+\cdots+a_{n}\right| \leqslant M ; \\
& \left|b_{0}-b_{1}\right| \leqslant c_{0}-c_{1}, \quad\left|b_{1}-b_{2}\right| \leqslant c_{1}-c_{2}, \ldots, \quad\left|b_{n}\right|<c_{n} ;
\end{aligned}
$$

then $\left|a_{0} b_{0}+a_{1} b_{1}+\cdots+a_{n} b_{n}\right| \leqslant M c_{0}$. In most applications we will have $b_{0}=c_{0}, b_{1}=c_{1}, \ldots, b_{n}=c_{n}$. Since the explicit form of $F_{K}(x)$ depends on the sign of $d$, we shall separately treat the two cases where $d<0$ and $d>0$ :

THEOREM 3. In the case where $d<0$ we have

$$
F_{K}(x)=\left(\frac{1}{2}\right) \sum_{1<n<|d|}(d / n) \mathbf{e}\left(i|d|^{-1 / 2} n x\right) /\left(1-\mathbf{e}\left(i|d|^{1 / 2} x\right)\right) ;
$$

the expression on the right-hand side represents a meromorphic function of $x$ with a simple pole at $m|d|^{-1 / 2} i$ and with $(4 \pi i)^{-1}(d / m)$ as the corresponding residue for every integer $m$ relatively prime to $d$.

Proof. By Abel's inequality we get

$$
\left|\sum_{k=n}^{N}(d / k) \mathbf{e}\left(i|d|^{-1 / 2} k x\right)\right|<|d| \mathbf{e}\left(i|d|^{-1 / 2} n x\right)
$$

for every $x>0$ and $N>n>1$; we also have

$$
\int_{0}^{\infty} \mathrm{e}\left(i|d|^{-1 / 2} n x\right) x^{s} d \log x=\left(2 \pi|d|^{-1 / 2} n\right)^{-s} \Gamma(s)
$$


for $\operatorname{Re}(s)>0$, and this tends to 0 as $n \rightarrow \infty$. Therefore

$$
F(x)=\left(\frac{1}{2}\right) \sum_{n=1}^{\infty}(d / n) \mathbf{e}\left(i|d|^{-1 / 2} n x\right)
$$

defines a continuous function $F$ on $\mathbf{R}_{+}^{\times}$. Furthermore $F(x) x^{s}$ for $\operatorname{Re}(s)>1$ is absolutely integrable with respect to $d \log x$ and for $\operatorname{Re}(s)>0$ the following calculation is legitimate:

$$
\begin{aligned}
\int_{0}^{\infty} F(x) x^{s} d \log x & =\left(\frac{1}{2}\right) \sum_{n=1}^{\infty}(d / n) \int_{0}^{\infty} \mathrm{e}\left(i|d|^{-1 / 2} n x\right) x^{s} d \log x \\
& =\left(\frac{1}{2}\right)\left(2 \pi|d|^{-1 / 2}\right)^{-s} \Gamma(s) L(s)=Z_{K}(s) .
\end{aligned}
$$

Finally $F(x)$ can be written as in the theorem and that expression represents an even meromorphic function of the complex variable $x$ with simple poles at most at $m|d|^{-1 / 2} i$ for $m=0, \pm 1, \pm 2, \ldots$; by Lemma 4 the corresponding residue is $(4 \pi i)^{-1}(d / m)$, hence $F(x)$ is holomorphic at $m|d|^{-1 / 2} i$ if $m$ is not relatively prime to $d$, e.g., for $m=0$. Therefore $F(x)$ coincides with $F_{K}(x)$. Q.E.D.

THEOREM 4. In the case where $d>0$ we have

$$
F_{K}(x)=\sum_{n=1}^{\infty}(d / n)\left(x^{2}+d^{-1} n^{2}\right)^{-1 / 2}
$$

the series on the right-hand side is uniformly convergent for $-\infty<x<\infty$ and for any $m \geqslant 1$ if we omit the first $m-1$ terms, then the remaining series in the complex variable $x$ is uniformly convergent for $|x|<m d^{-1 / 2}$ provided that we choose the square roots in the right-half plane.

Proof. We shall first recall the following fact: suppose that $F$ is a continuous function on $\mathbf{R}_{+}^{\times}$such that $F(x) x^{s}$ for $\operatorname{Re}(s)>\sigma_{0}$ is absolutely integrable with respect to $d \log x$ and that $Z(s)$ is its Mellin transform; put $\log x=2 \pi \xi$; then

$$
Z(s)=\int_{-\infty}^{\infty} 2 \pi x^{\sigma} F(x) \cdot \mathbf{e}(t \xi) d \xi
$$

for every $\sigma=\operatorname{Re}(s)>\sigma_{0}$. In other words $Z(s)$ considered as a function of $t$ is the Fourier transform of $2 \pi x^{\sigma} F(x)$ considered as a function of $\xi$. Therefore if $F_{1}(x), F_{2}(x)$ are two such functions with the additional property that their Mellin transforms $Z_{1}(s), Z_{2}(s)$ are absolutely integrable with respect to $d t$ for every $\sigma>\sigma_{0}$, then $Z_{1}(s) Z_{2}(s)$ is the Mellin transform of the convolutionproduct of $F_{1}(x), F_{2}(x)$.

After this remark we write $Z_{K}(s)=Z_{1}(s) Z_{2}(s)$, in which

$$
Z_{1}(s)=\frac{1}{2} \cdot \Gamma\left(\frac{1}{2} s\right), \quad Z_{2}(s)=\left(\pi d^{-1 / 2}\right)^{-s} \Gamma\left(\frac{1}{2} s\right) L(s) ;
$$

then in the above notation we will have 


$$
F_{1}(x)=\exp \left(-x^{2}\right), \quad F_{2}(x)=2 \cdot \sum_{n=1}^{\infty}(d / n) \exp \left(-d^{-1}(\pi n x)^{2}\right)
$$

with $\sigma_{0}=1$. This is clear for $F_{1}(x)$ and the verification for $F_{2}(x)$ is similar to the handling of $F(x)$ in the proof of Theorem 3; the absolute integrability of $Z_{1}(s), Z_{2}(s)$ with respect to $d t$ follows, e.g., from Lemma 2 . Therefore $Z_{K}(s)$ is the Mellin transform of

$$
F(x)=\int_{0}^{\infty} F_{1}(x y) F_{2}\left(y^{-1}\right) d \log y .
$$

We apply the classical Poisson formula to $F_{2}\left(y^{-1}\right)$; then by using Lemma 4 we get

$$
F_{2}\left(y^{-1}\right)=2 \pi^{-1 / 2} y \cdot \sum_{n=1}^{\infty}(d / n) \exp \left(-d^{-1}(n y)^{2}\right)
$$

hence

$$
F(x)=2 \pi^{-1 / 2} \cdot \int_{0}^{\infty}\left(\sum_{n=1}^{\infty}(d / n) \exp \left(-\left(x^{2}+d^{-1} n^{2}\right) y^{2}\right)\right) d y .
$$

As in the proof of Theorem 3 we can interchange the order of integration and summation and we get

$$
F(x)=\sum_{n=1}^{\infty}(d / n)\left(x^{2}+d^{-1} n^{2}\right)^{-1 / 2}
$$

We have so far assumed that $x>0$; by Abel's inequality this series is uniformly convergent for $-\infty<x<\infty$ and represents an even continuous function of $x$. Therefore $F(x)$ coincides with $F_{K}(x)$.

Finally we shall show that for every $m \geqslant 1$ if we omit the first $m-1$ terms, then the remaining series in the complex variable $x$ is uniformly convergent for $|x|<m d^{-1 / 2}$ (provided that we take positive square roots in the righthalf plane): for every $k>m$ and $|x|<m d^{-1 / 2}$ we put

$$
b_{k}=\left(x^{2}+d^{-1} k^{2}\right)^{-1 / 2}, \quad c_{k}=\left(d^{-1}\left(k^{2}-m^{2}\right)\right)^{-1 / 2}
$$

then $c_{k}$ tends to 0 as $k \rightarrow \infty$, and we have $\left|b_{k}\right|<c_{k}$. We also have

$$
\begin{aligned}
\left|b_{k}-b_{k+1}\right| & =\left|b_{k} b_{k+1}\left(b_{k}^{-1}+b_{k+1}^{-1}\right)^{-1}\right|\left(b_{k+1}^{-2}-b_{k}^{-2}\right) \\
& \leqslant c_{k} c_{k+1}\left(c_{k}^{-1}+c_{k+1}^{-1}\right)^{-1}\left(c_{k+1}^{-2}-c_{k}^{-2}\right)=c_{k}-c_{k+1} .
\end{aligned}
$$

Therefore by Abel's inequality we get

$$
\left|\sum_{k=n}^{N}(d / k)\left(x^{2}+d^{-1} k^{2}\right)^{-1 / 2}\right|=\left|\sum_{k=n}^{N}(d / k) b_{k}\right|<d \cdot c_{n}
$$

for every $N>n>m$; this implies the assertion. Q.E.D.

We note that the function $F_{K}(x)$ in Theorem 4 has an analytic (but 
nonmeromorphic) continuation to the whole $x$-plane with an algebraic singularity at $m d^{-1 / 2} i$ for every integer $m$ relatively prime to $d$.

Appendix (added in December of 1977). We shall make two remarks to take care of the referee's suggestions conveyed to us via Professor Shatz, the editor of the Transactions: the first remark concerns the relationship between this paper and a paper by M. Riesz in Acta Math. 40 (1916), 185-190 entitled Sur l'hypothèse de Riemann. In that paper Riesz proved the following formula:

$$
\Gamma(1-s) / \zeta(2 s)=\int_{0}^{\infty} F\left(x^{-1}\right) x^{s} d \log x,
$$

in which $F(x)$ is an entire function of $x$ with the following Maclaurin expansion:

$$
F(x)=-\sum_{n=1}^{\infty}(-x)^{n} / \Gamma(n) \zeta(2 n)
$$

he then showed that the Riemann hypothesis is equivalent to the growth condition $F(x)=O\left(x^{1 / 4+\delta}\right)$ as $x \rightarrow \infty$ for any $\delta>0$. And on p. 188 he stated that “ . . la méthode que nous avons suivie permet aussi d'exprimer la valeur réciproque d'une fonction quelconque représentée par une série de Dirichlet."

The above-recalled results of Riesz can be reproduced as follows: the known properties of $\Gamma(s)$ and $1 / \zeta(s)$ show that $\Gamma(1-s) / \zeta(2 s)$ for $\frac{1}{2}<$ $\operatorname{Re}(s)=\sigma<1$ is a continuous absolutely integrable function of $\operatorname{Im}(s)$ with absolutely integrable Fourier transform; hence if we denote its inverse Mellin transform by $F\left(x^{-1}\right)$, i.e., if we put

$$
F(x)=(1 / 2 \pi i) \int_{\sigma-\infty i}^{\sigma+\infty i} \Gamma(1-s) / \zeta(2 s) \cdot x^{s} d s,
$$

then by Fourier's inversion theorem we get (*). And by shifting the line of integration indefinitely to the right, we get the Maclaurin expansion of $F(x)$; the rest is as in Riesz's paper.

The above-outlined proof is simple enough that we can axiomatize the properties of $\Gamma(s)$ and $1 / \zeta(s)$ to formulate a general theorem. Riesz's paper and ours are closely related because both talk about the inverse Mellin transformation. However there is a remarkable difference: disregarding $\Gamma$-factors the function to be transformed is the inverse of a Dirichlet series in Riesz's case while it is not so in our case; the transformed function is a Schwartz function in our case, and that is precisely the point which we have emphasized, while it is not so in Riesz's case.

The second remark concerns possible generalizations. As far as a generalization of our paper is concerned, it was known to us when we submitted it. In the following we shall quote the mathematical part from two private letters both written on October 14, 1977: 
“... 'Theorem 1' can be generalized as follows: let $k$ denote an arbitrary number field and put

$$
\Delta_{k}(s)[\Phi]=\int_{A_{k}^{\times}} \Phi(x)|x|^{s} d^{\times} x ;
$$

take a special $\Phi$ of the form $\otimes_{v} \Phi_{v}$, in which $\Phi_{v}$ is the standard function for all $v$ except for one archimedean valuation, say $w$. Then for any finite algebraic extension $K$ of $k$ the equation

$$
\Delta_{k}(s)[\Phi]=\Lambda_{K}(s)
$$

is solvable by $\Phi_{w}$ in $\delta\left(k_{w}\right)$ if and only if $\zeta_{k}$ divides $\zeta_{K}$.

Theorem 1 has an exact analogue in the function-field case if $k$ has a prime divisor of degree 1 and if we take the corresponding valuation as $w$ and if we consider only those extensions $K$ of $k$ which have the same constant field as $k$. In both cases if we require that $\Phi_{w}\left(x_{w}\right)$ depend only on $\left|x_{w}\right|$, then it becomes unique. Furthermore in the number-field case if we exclude the classical case where $K=k$, then [the Maclaurin expansion of] this unique $\Phi_{w}$ is rarely analytic around 0 . In fact except for the case where $k=\mathbf{Q}$, $[K: k]=2$ treated in 'Theorem 2' and 'Theorem 3,' the only new case is the case where $k$ is imaginary quadratic (over $Q)$ and $[K: k]=2$; and in that case $\Phi_{w}\left(x_{w}\right)$ can be expanded into a series involving the modified Bessel function of the second kind of order $0 . "$

\section{REFERENCES}

1. H. Aramata, Ueber die Teilbarkeit der Dedekindschen Zetafunktionen, Proc. Imp. Acad. Japan 9 (1933), 31-34.

2. E. Artin, Ueber eine neue Art von L-Reihen, Abh. Math. Sem. Hamburg 3 (1924), 89-108.

3. R. Brauer, On the zeta-functions of algebraic number fields, Amer. J. Math. 69 (1947), 243-250.

4. G. H. Hardy and E. C. Titchmarsh, Self-reciprocal functions, Quart. J. Math. Oxford Ser. 1 (1930), 196-231.

5. E. Hecke, Ueber die Zetafunktion beliebiger algebraischer Zahlkörper, Göttingen Nachrichten 1917, pp. 77-89; Vandenhoeck \& Ruprecht, Göttingen, 1959, pp. 159-171.

6. J. Igusa, Complex powers and asymptotic expansions. I. Functions of certain types, Crelles J. Math. 268/269 (1974), 110-130.

7. H. Mellin, Abriss einer einheitlichen Theorie der Gamma- und der hypergeometrischen Funktionen, Math. Ann. 68 (1910), 305-337.

8. R. Nevanlinna, Eindeutige analytische Funktionen, Die Grundlehren der math. Wissenschaften, Band 46, Springer-Verlag, Berlin, 1936.

9. J. Tate, Fourier analysis in number fields and Hecke's zeta-functions, Thesis, Princeton University, 1950; Algebraic Number Theory, Thompson, Washington, D.C., 1967, pp. 305-347.

10. H. Weber, Lehrbuch der Algebra. III, Friedr. Vieweg, Braunschweig, 1908.

11. A. Weil, Sur les "formules explicites" de la théorie des nombres premiers, Comm. Sém. Math. Univ. Lund, 1952, pp. 252-265.

12. ___ Fonction zeta et distributions, Séminaire Bourbaki, vol. 312, 1966, pp. 1-9.

Department of Mathematics, The Johns Hopkins University, Baltimore, Maryland 21218

Current address: 14209 Greencroft Lane, Cockeysville, Maryland 21030 